\title{
Chitosan films promote formation of olfactory neurospheres and differentiation of olfactory receptor neurons*
}

\author{
Sheng-Tien Li ${ }^{1}$, Tai-Horng Young ${ }^{1}$, Chi-Te Wang ${ }^{3}$, Tsung-Wei Huang 2,3,4 \\ Rhinology 56: 4, 336-342, 2018 \\ https://doi.org/10.4193/Rhin17.155 \\ 'Institute of Biomedical Engineering, College of Medicine and College of Engineering, National Taiwan University, No. 1, Sec. 1 Jen- \\ Ai Road, Taipei 100, Taiwan \\ *Received for publication: \\ 2 Department of Electrical Engineering, College of Electrical and Communication Engineering, Yuan Ze University, Taoyuan, Taiwan July 17, 2017 \\ ${ }^{3}$ Department of Otolaryngology, Far Eastern Memorial Hospital, Taipei, Taiwan \\ Accepted: April 26, 2018 \\ ${ }^{4}$ Department of Health Care Administration, Oriental Institute of Technology, Taipei, Taiwan
}

Background: Olfactory dysfunction significantly impairs the life quality of patients. Therefore, a model needs to be developed for anosmia. Chitosan is a biodegradable natural polysaccharide that has been widely studied for regenerative purposes in the nervous system. However, whether chitosan promotes differentiation of olfactory receptor neurons or regulates formation of neurospheres in the olfactory system remains unexplored.

Methodology: Olfactory neuroepithelial cells were isolated from embryonic wistar rats on day 17, and cultured on controls and chitosan films for 12 days. The effects of treatment were assessed using immunocytochemistry, quantitative polymerase chain reaction and western blots following culturing. The substrate of poly-L-lysine-co-laminin was adopted as a control.

Results: In contrast to the flat layer on controls, olfactory neuroepithelial cells form olfactory neurospheres on chitosan films with steadily increasing diameter. The olfactory neurospheres contain basal cells, as well as immature and mature olfactory receptor neurons. The expression level of olfactory marker protein is higher on chitosan films than those on controls in gene and protein levels, and the olfactory transduction elements also express a similar trend. Mature olfactory receptor neurons are found predominantly at the periphery of the olfactory neurospheres.

Conclusions: Chitosan films not only facilitate formation of olfactory neurospheres, but also promote differentiation of olfactory receptor neurons. Chitosan is a potential biomaterial to establish an in vitro culture model to treat olfactory dysfunction in future.

Key words: chitosan; anosmia; olfactory receptor neurons; olfactory mucosa

\section{Introduction}

Olfactory dysfunction significantly affects quality of life by altering appetite, reducing the ability to identify nearby dangerous situations, and even resulting in depression ${ }^{(1,2)}$. Hyposmia varies in the general population from $13 \%$ to $18 \%$, and anosmia from $4 \%$ to $6 \%{ }^{(3)}$. In the olfactory system, the olfactory neuroepithelium (ON) operates the first relay point of odor sensation. The mammalian $\mathrm{ON}$, which harbors olfactory receptor neurons (ORNs), is located in the nasal cavity, while this neuroepithelium is split into three compartments, basal, middle and apical. The basal compartment is close to the basal lamina of the epithelium, and consists of basal cells that express the achaete-scute family bHLH transcription factor 1 (Ascl1); the middle compartment contains immature and mature ORNs that individually express $\beta$ III tubulin and olfactory marker protein (OMP), and the apical layer of the ON comprises sustentacular cells ${ }^{(4)}$. Particularly, unlike other cranial neurons, ORNs can be continuously replaced with new ones during normal neuronal turnover and environmental insults ${ }^{(5)}$. However, this unique ability deteriorates with age and in response to various toxic factors, including smoking, viruses, inflammation, medication and environmental factors, leading to olfactory dysfunction ${ }^{(6,7)}$. Although many approaches, including vitamin A, zinc and various growth factors, have promising findings, they have not been applied as clinical practice methods for olfactory dysfunction ${ }^{(7-10)}$. Topical or systemic application of glucocorticoids is a common clinical treatment, but its therapeutic effect is controversial. Oral steroids can improve olfaction in sinonasal disease, but the reduced sense 
of smell tends to recur shortly after the treatment is stopped ${ }^{(11)}$. Topical steroids are well tolerated and are effective treatments of olfactory loss associated with severe nasal and sinus disease ${ }^{(12)}$. However, it appears to have a minor effect on olfactory dysfunction in some reports, especially when considering longterm changes ${ }^{(13,14)}$.

A therapeutic trend is transplantation and engraftment of basal cells in the damaged $\mathrm{ON}{ }^{(15)}$. The $\mathrm{ON}$ can be regenerated using autologous olfactory basal cells to repair damage, and to alleviate the consequent dysfunction ${ }^{(16)}$. Hence, an in vitro culture system that can encourage olfactory neuronal differentiation and expansion of basal cells is essential for the regeneration of ON. Among culture conditions, biomaterials play a crucial role in mediating cellular morphology, proliferation and differentiation. Chitosan is a cationic polysaccharide with a variable number of randomly located D-glucosamine and $\mathrm{N}$-acetyl-glucosamine groups ${ }^{(17)}$. This biopolymer has been demonstrated to bridge large gaps in the peripheral nerves with chitosan-coated silicone tubes. Chitosan is also reported to regulate formation of neurospheres ${ }^{(18-20)}$. Compared with the adherent culture, this sphere formation in vitro culture has numerous multipotent progenitors to facilitate incorporation into host epithelium, which is a requirement for eventual translational use ${ }^{(21)}$. Therefore, neurospheres can serve as biomarkers for engraftment capacity. However, whether chitosan can promote differentiation of olfactory neuroepithelial cells (ONCs) or regulate formation of olfactory neurospheres remains unexplored. The objective of this study is to evaluate the effect of chitosan films on development and differentiation of ONCs in an in vitro culture system.

\section{Materials and methods}

\section{Cell preparation and culture}

All experimental procedures and animals used were approved by the National Taiwan University Animal Care and Use Committee (agreement number: 20140357). Briefly, the ON was obtained from embryonic wistar rats on day 17 (E17) using the protocol in our previous study ${ }^{(22)}$. Briefly, the ON was cut into small pieces in cold Hank's balanced salt solution and then digested with $0.125 \%$ Trypsin/EDTA for 30 minutes at $37^{\circ} \mathrm{C}$. After digestion, the dissociated cells were collected by centrifuge and resuspended in DMEM-F12 (serum-free) containing $20 \mathrm{ng} / \mathrm{mL}$ bFGF, $20 \mathrm{ng} / \mathrm{mL}$ EGF, 2\% B27 and 1\% penicillin/streptomycin. The half medium was added on alternate days. For the experiment, harvested cells were seeded on tissue culture plates pre-coated with poly-L-lysine-co-laminin (PLL-La) or on chitosan films at $37^{\circ} \mathrm{C}$ in a humidified $5 \% \mathrm{CO}_{2}$ and $95 \%$ air atmosphere.

\section{Preparation of substrate-coated culture plates}

Substrate-coated was coated on tissue culture plates according to a protocol detailed previously ${ }^{(23)}$. Briefly, culture plates were pretreated with PLL (P2636, Sigma-Aldrich, St. Louis, MO, USA)
Table 1. List of gene-specific primers used for qPCR assay.

\begin{tabular}{|c|c|c|}
\hline Gene & Sequence & Product size \\
\hline Ascl1 & $\begin{array}{l}\text { ACTTGAACTCTATGGCGGGTT } \\
\text { TGCTTCCAAAGTCCATTCCCA }\end{array}$ & $151 \mathrm{bp}$ \\
\hline Blll tubulin & $\begin{array}{l}\text { ATGAAGGAGGTGGATGAGCAG } \\
\text { TTGCCGATGAAGGTGGACGA }\end{array}$ & $102 \mathrm{bp}$ \\
\hline$O M P$ & $\begin{array}{l}\text { CTTGATTTCATCCAGCAGCA } \\
\text { ATGAGGTTGGTGAGGTCTGG }\end{array}$ & $122 \mathrm{bp}$ \\
\hline$G_{\text {olf }}$ & $\begin{array}{l}\text { TTGACCACGTGAAGAAGCTG } \\
\text { TCCTGGTCTGTGGGTGTGTA }\end{array}$ & $148 \mathrm{bp}$ \\
\hline$A D C Y 3$ & $\begin{array}{l}\text { CGTGGTCTCCTGTGTTGTGC } \\
\text { CGCACAGGTAGAGGAAGACG }\end{array}$ & $124 \mathrm{bp}$ \\
\hline GAPDH & $\begin{array}{l}\text { GGCTGCCTTCTCTTGTGACA } \\
\text { CCGTGGGTAGAGTCATACTGGA }\end{array}$ & $101 \mathrm{bp}$ \\
\hline
\end{tabular}

at a concentration of $52 \mu \mathrm{g} \mathrm{cm}^{-1}$. Then, $2 \mu \mathrm{g} \mathrm{cm}^{-1}$ laminin was coated on top of the PLL. The culture plates were then put in the incubator at $37^{\circ} \mathrm{C}$ until their use at the same day. The excessive laminin was removed immediately before seeding ONCs. This group was adopted as controls.

\section{Preparation of chitosan films culture plates}

Chitosan was coated on tissue culture plates using methods described in a previous study, with some modifications ${ }^{(20)}$. Briefly, $0.5 \mathrm{~mL}$ 1\% (w/w) chitosan solution (C-3646, Sigma-Aldrich) dissolved in $0.5 \mathrm{~N}$ acetic acid was added into each well of six-well plates and evaporated in a convection oven at $60^{\circ} \mathrm{C}$ until the casting solution became solid. Then, the nascent films were neutralized using $0.5 \mathrm{~N} \mathrm{NaOH}$. Finally, these films were sterilized in $70 \%$ alcohol under ultraviolet light, and rinsed extensively with distilled water, before being used for cell culture.

\section{Morphological examination}

Cell morphology was observed under an inverse phase contrast microscope (TS-100, Nikon, Tokyo, Japan) and scanning electron microscope (S-4800, Hitachi, Tokyo, Japan). The diameter and projected area of spheroids were calculated using the software Image $J$ in ten randomly selected fields for each sample. Only those with the diameter of more than $30 \mu \mathrm{m}$ were considered spheroids. Ratio of olfactory neurosphere projected area was defined by a percentage of projected area of neurosphere in a view.

Reverse transcription- quantitation polymerase chain reaction (RT-qPCR)

Total RNA was extracted from the cultured cells using a Trizol reagent (Invitrogen, CA, USA). The synthesis of first-strand cDNA was performed by the protocol from High-Capacity CDNA Reverse Transcription Kits (Applied Biosystems, Foster City, CA, USA). The CDNA was applied as the template for the qPCR assay 


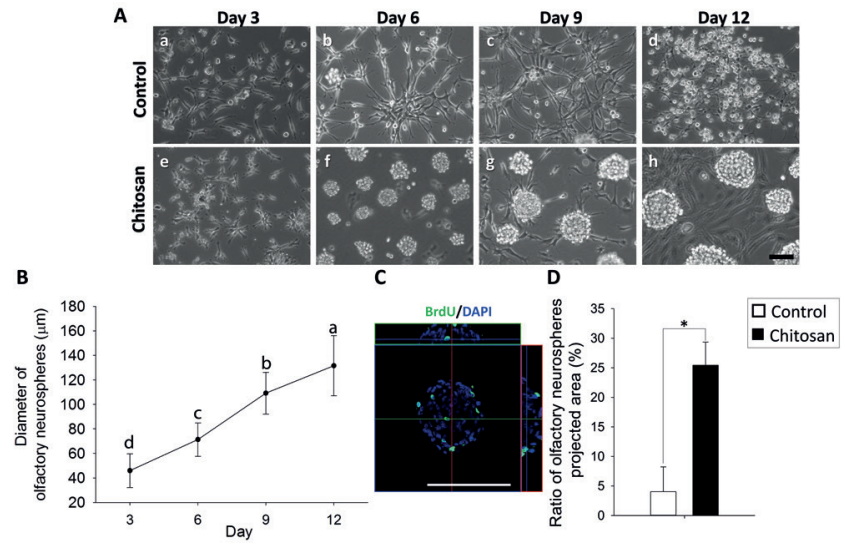

Figure 1. The morphological changes in primary ONCs on controls (PLLLa) (a-d) and chitosan films (e-h) after day 3, day 6, day 9 and day 12 (A). Diameters of olfactory neurospheres on chitosan films change from day 3 to day 12 (B). Proliferating cells within the spheres are identified with anti-BrdU (green), and counterstained with DAPI to visualize the nuclei (C). The ratio of projected area of olfactory neurospheres at day 12 (D). Results are expressed as means \pm SD. Different letters $(a, b, c, d)$ and asterisk indicate $p<0.05$. Scale bar $=100 \mu \mathrm{m}$.

of specific gene expression performed with the gene-specific primers listed in Table 1. The gene expression level was analyzed and normalized to GAPDH for each cDNA sample. Relative quantity of gene expression was calculated with the expression levels each group divided by those in controls at day 6 .

\section{Immunocytochemical staining}

The samples were fixed with $4 \%$ paraformaldehyde and permeabilized with $0.1 \%$ Triton X-100 (X100; Sigma-Aldrich) for 10 minutes at room temperature. After fixing, the samples were blocked in $3 \%$ bovine serum albumin for 20 minutes, and incubated with primary antibodies at $4^{\circ} \mathrm{C}$ overnight. The primary antibodies and their dilution utilized were used in this study: anti-Ascl1 (Ab74065; 1:1000; Abcam, Cambridge, UK), anti-ßIII tubulin (Ab1 18627; 1:1000; Abcam), anti-OMP (ab62144, 1:100; Abcam), anti-OMP (NB110-74751, 1:100; Novus, CO, USA), anti-olfactory neuron specific-G protein (Golf) (GTX110520; 1:100; GeneTex, CA, USA), anti-adenylate cyclase type 3 (ADCY3) (Ab125093; 1:1000; Abcam) and anti-5-bromo-2'-deoxiuridine (BrdU) (MAB3424; 1:1000; Millipore, Billerica, MA, USA). The samples were then washed and incubated with Alexa Fluor 488 and/or Alexa Fluor 555-conjugated secondary antibodies, and counterstained with DAPI. Particularly, before day 6, cells were incubated with $10 \mu \mathrm{M}$ BrdU for 24 hours to identify S-phase cells since BrdU, a thymidine analog, would incorporate into the newly synthesized DNA in replicating cells. Images were taken with Leica DMI600 microscope and a confocal microscope (LSM510, Carl Zeiss, Germany). Further, the BrdU-positive cells within olfactory neurospheres were counted and divided by the total
Day 6
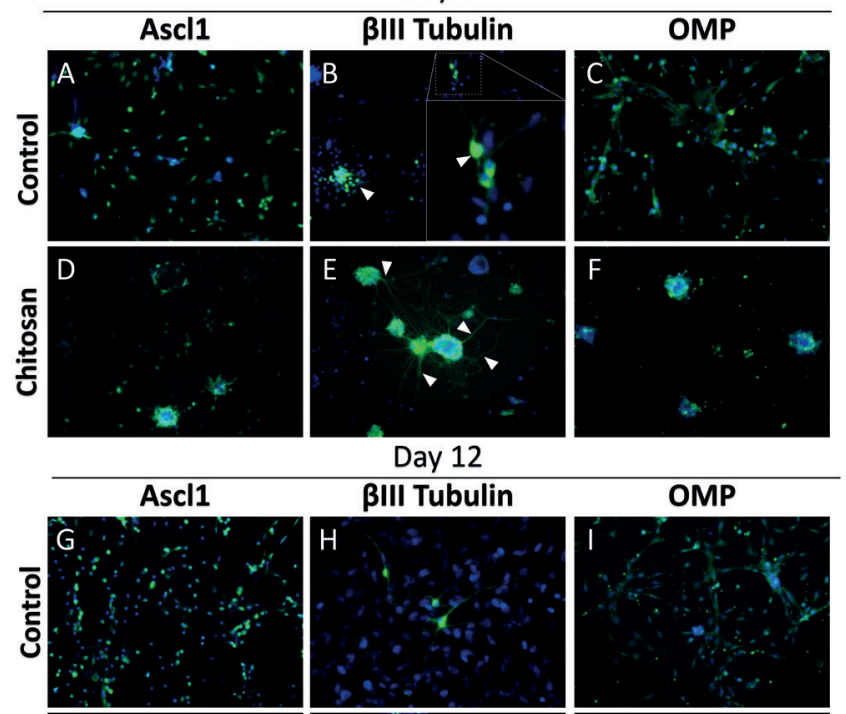

BIII Tubulin
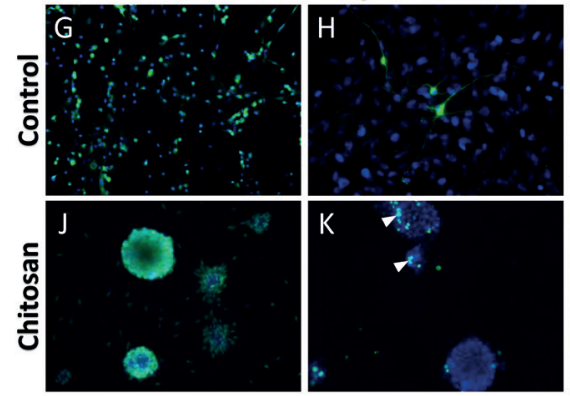

OMP

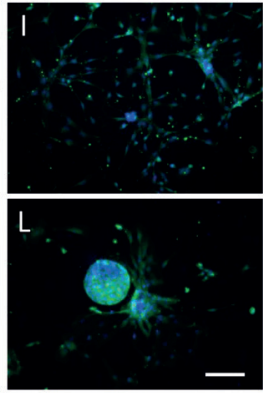

Figure 2. Immunocytochemistry of basal cells, immature ORNs and mature ORNs at day 6 (A-F) and day $12(\mathrm{G}-\mathrm{L})$, on controls and chitosan films. Basal cells, immature ORNs and mature ORNs are respectively labeled by anti-Ascl1, anti-BIII Tubulin and anti-OMP antibodies. They are identified with Dylight488 (green). Nuclei are labeled with DAPI (blue). $\beta$ III Tubulin is clearly present throughout the neuron, in soma, dendrites and axons. Arrows indicate $\beta$ III Tubulin-positive cells. Scale bar $=100 \mu \mathrm{m}$ for all views.

number using ImageJ in at least ten randomly selected fields.

\section{Western blot analyses}

Each lysate was obtained from individual culture wells using a radioimmunoprecipitation assay lysis buffer containing protease inhibitor cocktail (Roche Diagnostics, Indiana, IN, USA) to avoid protein degradation. The protein samples were then denatured and separated by $12 \%$ SDS-PAGE gels, and blotted onto PVDF membranes (Millipore, Billerica, MA. USA). After blocking in a CISblocking buffer (CIS-Biotechnology, Taiwan) at room temperature for 60 seconds, the membranes were probed with the specific primary antibody at $4^{\circ} \mathrm{C}$ overnight, washed, incubated in horseradish peroxidase (HRP)-conjugated secondary antibodies, and finally visualized using enhanced chemiluminescence (ECL; Millipore). The antibodies used in this study were antiAscl1 (Ab74065; 1:1000), anti-ßlll tubulin (Ab118627; 1:1000), anti-OMP (Ab98124; 1:1000), anti-olfactory neuron specific-G protein (Golf) (Ab74049; 1:1000) and anti-adenylate cyclase type 3 (ADCY3) (Ab125093; 1:5000) (all from Abcam, Cambridge, MA, USA). After detachment of previous primary antibodies, the membranes were also probed with GAPDH antibodies, 

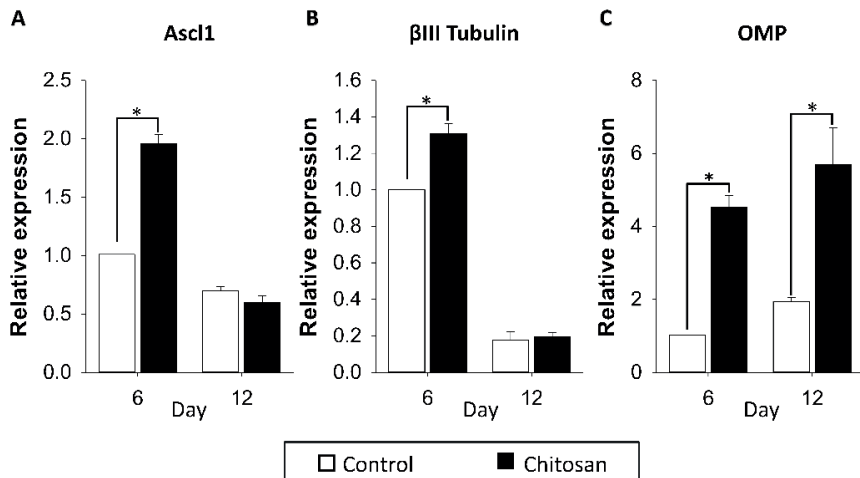

$\square$ Control

Chitosan

Figure 3. RT-qPCR measurements for marker genes of basal cells, immature ORNs and mature ORNs, on controls and chitosan films. The expression of Ascl1 mRNA (A), BIII Tubulin mRNA (B), and OMP mRNA (C) in ONCs at day 6 are higher on chitosan films than on controls. Results are expressed as means \pm SD. Asterisk indicates $p<0.05$.

which acted as an internal control. Immunoreactive bands were quantified using Image J.

\section{Measurement of calcium transients}

Olfactory neurospheres cultured on chitosan films at day 12 were plated on gelatin-coated $35 \mathrm{~mm}$ coverslips for 1 day prior to testing. Briefly, cells were loaded with $1 \mu \mathrm{M}$ fura-2 AM (F-1225, Invitrogen) and $20 \mu \mathrm{g} / \mathrm{ml}$ Pluronic ${ }^{\oplus} \mathrm{F}-127$ (P3000MP, Invitrogen) at room temperature for 30 minutes in a culture medium, followed by 2 rinses with Hank's Balanced Salt Solution containing HEPES for 30 minutes to cleave the excess fura-2 AM. A fura2loaded cell was then respectively exposed to the alternating excitation wavelengths of $340 \mathrm{~nm}$ and $380 \mathrm{~nm}$ for $\mathrm{Ca}^{2+-}$ bound and $\mathrm{Ca}^{2+}$-free dyes by lonOptix system (IonOptix, Milton, MA, USA). A solution of $300 \mu \mathrm{M} 3$-isobutyl-1-methylxanthine (IBMX, Invitrogen) plus $10 \mu \mathrm{M}$ forskolin (FSK) acted as a stimulator to induce intracellular calcium transients, according to a previous protocol ${ }^{(23)}$. The calcium waves were described using the ratio $340 / 380$. All parameters were analyzed off-line using lonWizard (IonOptix, Milton, MA, USA).

\section{Statistical analysis}

The results were observed at least three independent experiments, with the data expressed as mean \pm standard deviation (SD). Statistical significance was evaluated using paired t-test or one way ANOVA followed by Tukey's post-hoc test with $p<0.05$ being considered significant.

\section{Results}

Morphology of ONCs on chitosan films vs. controls (PLL-La) Cells undergo morphological changes after contact with biomaterials to stabilize the cell-substrate interface, which plays a significant role in regulating subsequent cell behaviors such as
A
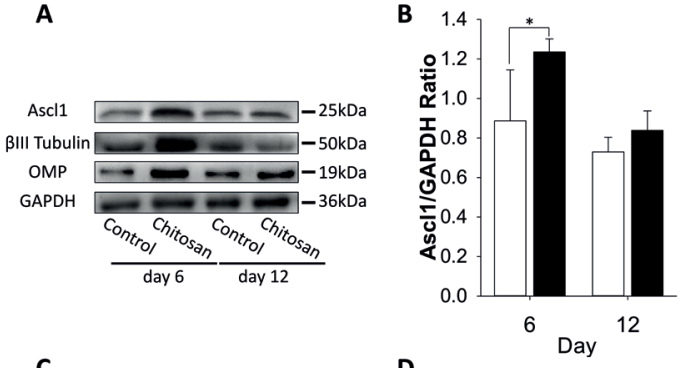

C
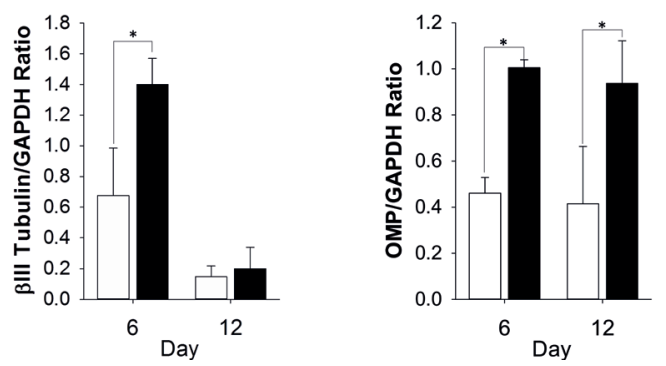

Figure 4. Western blot analyses of Ascl1, $\beta$ III Tubulin, OMP and GAPDH proteins in ONCs (A). The expression of $\beta$ III Tubulin and OMP protein in ONCs are significantly higher on chitosan films than on controls at day 6 . Ascl1/GAPDH ratio (B). Blll Tubulin/GAPDH ratio (C). OMP/GAPDH ratio (D). Results are expressed as means \pm SD. Asterisk indicates $p<0.05$.

survival, proliferation and differentiation. In controls, cells with fusiform-like morphology attached to the substrate and extended neurites at day 3 (Figure 1A (a)). Fusiform cells in this group were variable in size and shape, and lengthened neurites over time. The ONCs reached approximately $80 \%$ confluence until day 9 , and retained flat and bipolar-like morphology. Lots of round cells appeared at day 12 (Figure $1 \mathrm{~A}(\mathrm{~d})$ ). Conversely, ONCs cultured on chitosan films exhibited small neurospheres by day 3 (Figure 1A (e)). Small colony-like structures were sometimes formed. The number and size of those neurospheres gradually rose after day 6 , while maintaining semi-attachment to chitosan films. The diameter of olfactory neurospheres steadily increased from $46 \mu \mathrm{m} \pm 13.6 \mu \mathrm{m}$ at day 3 to $132 \mu \mathrm{m} \pm 24.5 \mu \mathrm{m}$ at day 12 , and BrdU was also expressed within olfactory neurospheres $(7.5 \% \pm$ $2.7 \%$, Figures $1 \mathrm{~B}-\mathrm{C}$ ). BrdU, an analog of thymidine, labels newly synthesized DNA in mitotic cells, and is commonly used in the detection of proliferating cells. Additionally, this projected area covered by neurospheres was larger on chitosan films than on controls. The percentages of the projected area on chitosan films were $25 \% \pm 3.9 \%$, compared to $4 \% \pm 4.1 \%$ on controls at day 12 , showing a significant difference $(p<0.05)$ (Figure1D).

The ORN differentiation of ONCs on chitosan films vs. controls

ONCs have been well characterized for stage-specific markers ${ }^{(4)}$. In the ONC development, a marker of basal cells, Ascl1, plays a crucial choice point at an early stage in the ORN lineage to initiate a differentiation program. OMP is a prototypical form, and is 


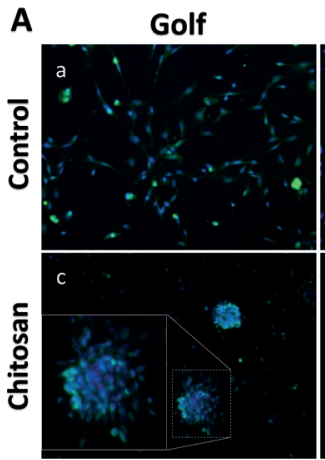

D

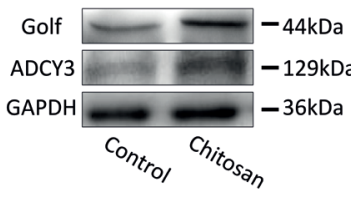

ADCY3

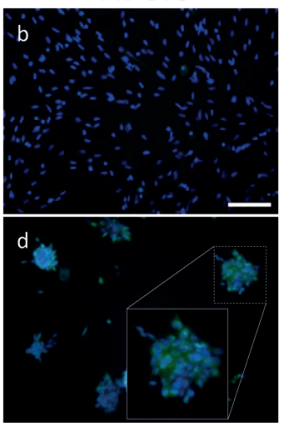

E

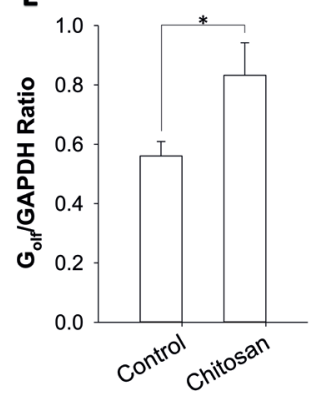

B

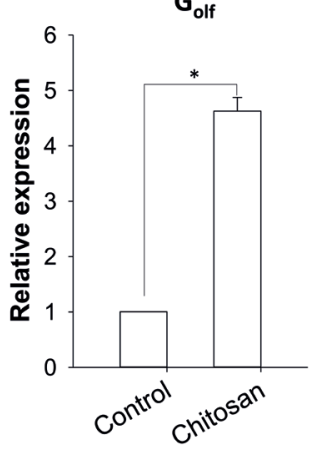

$\mathbf{F}$

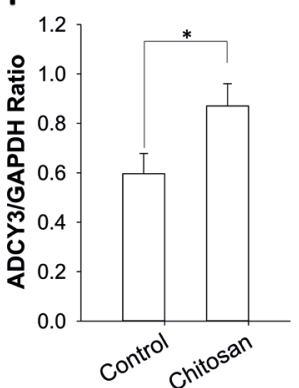

C

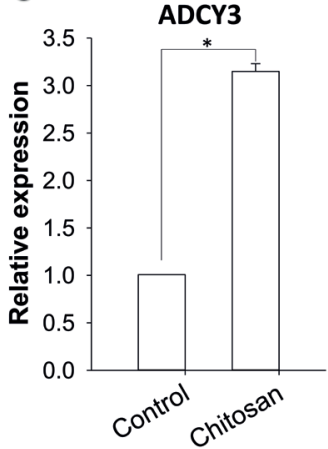

G

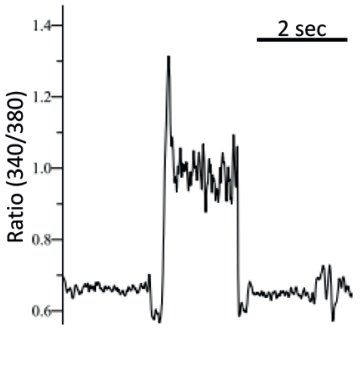

Figure 5. Immunocytochemical detection of Golf and ADCY3 on controls (a-b) and chitosan films (c-d) (A). ONCs are immunostained at day 12, and identified with Dylight488 (green). Nuclei are labeled with DAPI (blue). The micrographs show that Golf and ADCY3 are respectively located in cell bodies and membranes. RT-qPCR measurements for functional elements on controls and chitosan films. ONCs on chitosan films have significantly higher levels than controls of Golf mRNA (B) and ADCY3 mRNA (C). Western blot analyses of Golf, ADCY3 and GAPDH proteins in ONCs at day 12 (D). Golf/GAPDH ratios (E). ADCY3/GAPDH ratios (F). The expressions of Golf and ADCY3 protein in ONCs are significantly higher on chitosan films than on controls. Fura-2-loaded olfactory neurospheres are stimulated with FSK + IBMX, and the perfusion time of stimulators is indicated by the horizontal bars above the traces (G). Scale bar $=100 \mu \mathrm{m}$. Results are expressed as means \pm SD. Asterisk indicates $p<0.05$.

expressed almost exclusively in mature ORNs, while $\beta$ III tubulin is a neuron-specific isoform of tubulin, and is widely accepted as a marker of immature ORNs. At day 6, immunofluorescence revealed that ONCs on both groups expressed one of these stage-specific markers, Ascl1, $\beta$ III tubulin and OMP (Figure 2). $\beta I I I$ tubulin was clearly present throughout the neuron, in soma, dendrites and axons (Figure 2E). At day 12, the expression level of $\beta$ III tubulin significantly fell on both groups (Figs. 2H \& 2K), but the expression level of OMP was higher on chitosan films than on controls (Figures $2 \mathrm{~L} \& 2 \mathrm{l}$ ). The distribution of mature ORNs with positive OMP gathered at spheroid cells was also noteworthy (Figure $2 \mathrm{~L}$ ). Further evaluation of gene expression with RT-qPCR revealed $2.0 \pm 0.1$ fold upregulated Ascl 1 and $1.3 \pm 0.1$ fold upregulated $\beta$ III tubulin genes on chitosan films comparing to those on controls at day 6 (Figures 3A \& 3B, p < 0.05). Notably, the mRNA ratio of OMP relative to the internal gene GAPDH was significantly higher on chitosan films than on controls $(5.7 \pm 1.0$ fold) at day 12 (Figure 3C, $p<0.05$ ). Western blot analyses of Ascl1, $\beta$ III tubulin and OMP further confirmed the results (Figure 4).
Olfactory transduction elements of ORNs on chitosan films vs. controls

To further determine whether ORNs have functional characteristics in these cultures, the levels of Golf and ADCY 3 were measured, as these are essential elements and hallmarks of functionally mature ORNs (24). Immunofluorescence revealed at day 12 that ORNs on chitosan films expressed more Golf and $A D C Y 3$ than those on controls (Figure 5A). The Golf and $A D C Y 3$ were respectively found throughout their cell bodies and membranes, in accordance with previous reports (25) (Figure 5A, micrographs). The chitosan films and controls had significantly different immunofluorescence patterns. The distribution of Golf- and ADCY3-positive cells also gathered at spheroid cells. In comparison to controls, the gene expression level of ONCS on chitosan films exhibited significant upregulation of Golf and ADCY3 genes at day 12, at 4.6-fold and 3.2-fold respectively (Figures 5B \& 5C, p < 0.05). Western blot analyses of Golf and $A D C Y 3$ further confirmed these findings (Figure5D-F). Particularly, the olfactory neurospheres showed a typical response peak of intracellular calcium in response to IBMX+FSK, suggesting that olfactory neurospheres cultured on chitosan films would remain functional ORNs (Figure 5G). 


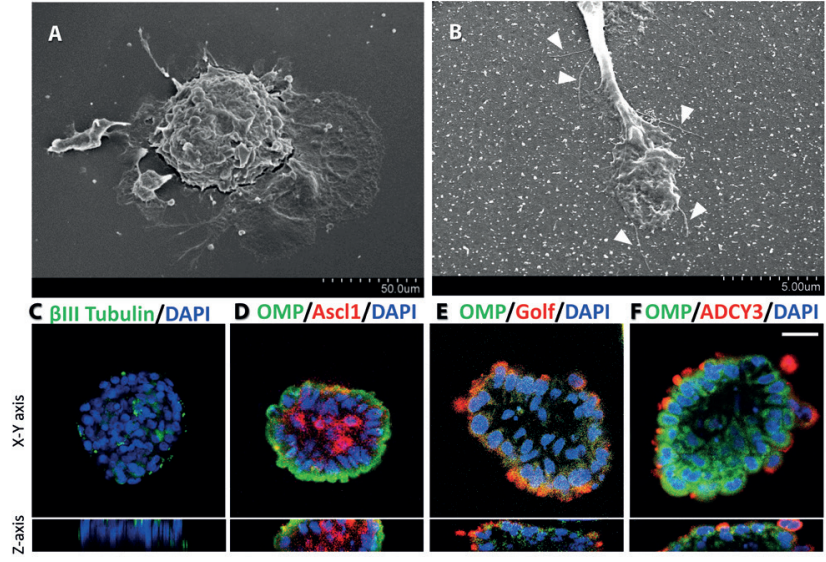

G

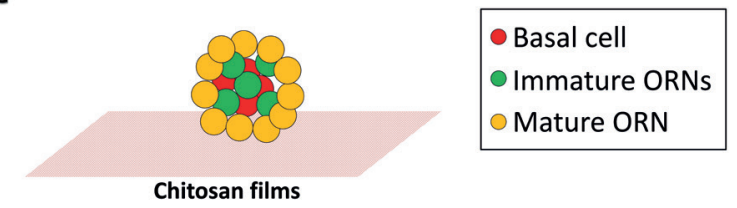

Figure 6. Scanning electron micrographs of olfactory neurospheres on chitosan films at day 6 (A). (Magnification $\times 600$ ). The small population of bipolar neurons on chitosan films has fine filamentous extensions from their terminal knobs. They are cilia-like structures ( $B$, arrow). (Magnfication $\times 6000$ ). Representative confocal images show cellular composition of chitosan-expanded olfactory neurospheres. Photographs of sectioned olfactory neurospheres show $\beta$ III Tubulin (green, C) and double staining for OMP (green) with Ascl1 (red, D), Golf (red, E) or ADCY3 (red, F). Nuclei are labeled with DAPI (blue). Diagram of the cellular distribution within olfactory neurospheres (G). Scale bar $=20 \mu \mathrm{m}$.

\section{Analysis of olfactory neurospheres}

Apparent neurospheres appeared at day 6, with an average diameter of $71.3 \mu \mathrm{m} \pm 13.7 \mu \mathrm{m}$. The small population of multicellular spheroids on chitosan films had fine filamentous extensions from their terminal knobs. They are cilia-like structures (Figures $6 \mathrm{~A} \& 6 \mathrm{~B})$. Three-dimensional analyses of olfactory neurospheres with confocal microscopy revealed that basal cells, expressing Ascl1, gathered in the center of neurospheres, while the ORNs, expressing $\beta$ III tubulin or OMP, were predominantly distributed at the periphery (Figures $6 \mathrm{C} \& 6 \mathrm{D}$ ). Double staining of the olfactory transduction elements and OMP showed that the expressions of Golf and ADCY3 were co-localized with OMP at the periphery (Figures $6 \mathrm{E} \& 6 \mathrm{~F}$ ). The distribution of immature and mature ORNs in the olfactory neurospheres was similar to in vivo ON (Figure 6G).

\section{Discussion}

Several groups of researchers have developed primary culture systems derived from $\mathrm{ON}$ in embryonic, neonatal or adult murine to study neurogenesis in the past two decades ${ }^{(23,26)}$. In particular, the ON still contains many immature ORNs that are able to further mature in vitro at the E17 stage ${ }^{(27)}$. According to our previous study, these primary ONCs simultaneously expressed markers of horizontal basal cells and proliferation, i.e. cytokeratin 5 and BrdU, at day $3^{(22)}$. Among substrates adopted as controls, PLL has a significantly positive effect on proliferation of ONCs, and laminin is an extracellular matrix within the ON. Both substrates not only improve axon outgrowth of ORNs and its pathfinding in the olfactory system, but are also widely used as substrates in ONC cultures ${ }^{(23,28)}$. Therefore, this investigation harvested rat ONCs at E17, and utilized PLL-La as a substrate for comparison ${ }^{(23)}$. As depicted in Figure 1A (a-d), ONCs can proliferate on controls with variable morphology to reach confluence after 12 days. However, in comparison to the two-dimensional monolayer culture, providing more physiologically relevant information and creating an in vivo similarity in three-dimension culture systems have many advantages, which may benefit treatment of olfactory dysfunction in the future.

Chitosan has been widely utilized in tissue engineering of neurological and nasal systems, as it bridges large gaps in the peripheral nerves with chitosan-coated silicone tubes, and exhibits permeation-enhancing properties for mucosal drug delivery ${ }^{(18,29,30)}$. Although several reports have demonstrated that chitosan films can promote formation of spheres ${ }^{(19,20)}$, no reports have considered whether chitosan promotes formation of ONCs. This work found that ONCs cultured on chitosan films form olfactory neurospheres, in contrast to the almost flat layer on controls (Figure 1A). Cells within the olfactory neurospheres express the markers of basal cells and proliferation, i.e. Ascl1 and BrdU (Figure 6D \& Figure 1C). The expression of BrdU within olfactory neurospheres explains why the diameter of spheres steadily rises during culture periods (Figure $1 \mathrm{~B}-\mathrm{C}$ ). These findings indicate that chitosan can promote formation of olfactory neurospheres with numerous basal cells, which may be valuable for translational purpose in the future.

Another important issue is whether chitosan promotes differentiation and maturation of ORNs. This work indicates that ONCs express more terminal differentiation markers of ORNs, i.e. OMP, Golf and ADCY3 on chitosan films in gene and protein levels than on controls, implying a significantly greater number of mature ORNs on chitosan films. Additionally, many olfactory neurons develop cilia-like structures extending from the process of an ORN (Figure 6B). The confocal microscope analyses of olfactory neurospheres reveal that mature ORNs expressing OMP, Golf and ADCY3 are co-localized within the out-layer of the spheroids (Figure 6D-F). This finding indicates that spheroids may start to develop polarity and behave like their in vivo counterparts ${ }^{(31)}$ (Figure 6G).

\section{Conclusion}

This investigation is the first to show that chitosan promotes 
formation of olfactory neurospheres that not only maintain proliferation of basal cells, but also enhance ORN maturation. However, whether these olfactory neurospheres can be applied to facilitate $\mathrm{ON}$ regeneration in vivo needs further investigation.

\section{Acknowledgement}

The authors would like to thank the Ministry of Science and Technology of Taiwan and Far Eastern Memorial Hospital for financially supporting this research under Contract No. MOST104-2314-B-418-011-MY3 and FEMH-2017-C-016.

\section{Authorship contribution}

STL: study design, acquisition and interpretation of data, drafting of the manuscript, final approval of the version, accountability for all aspects of the work. THC, CTW, and TWH: study design, acquisition and interpretation of data, revision of the manuscript, final approval of the version, accountability for all aspects of the work.

\section{Conflict of interest}

The authors declared that no conflict of interest exists.

\section{References}

1. Doty RL. The olfactory system and its disorders. Seminars in neurology. 2009; 29(1): 74-81.

2. Scangas GA, Bleier BS. Anosmia: Differential diagnosis, evaluation, and management. Am J Rhinol Allergy. 2017; 31(1): 3-7.

3. Philpott CM, Boak D. The impact of olfactory disorders in the United kingdom. Chemical senses. 2014; 39(8): 711-718.

4. Kam JW, Raja R, Cloutier JF. Cellular and molecular mechanisms regulating embryonic neurogenesis in the rodent olfactory epithelium. Int J Dev Neurosci. 2014 Oct;37:76-86.

5. Duggan CD, Ngai J. Scent of a stem cell. Nature neuroscience. 2007; 10(6): 673-674.

6. Doty RL, Shaman P, Applebaum SL, Giberson R, Siksorski L, Rosenberg L. Smell identification ability: changes with age Science (New York, NY). 1984; 226(4681): 1441-1443.

7. Hummel T, Whitcroft KL, Andrews $P$, et al. Position paper on olfactory dysfunction. Rhinol Suppl. 2017 Mar;54(26):1-30.

8. DeHamer MK, Guevara JL, Hannon K, Olwin BB, Calof AL. Genesis of olfactory receptor neurons in vitro: regulation of progenitor cell divisions by fibroblast growth factors. Neuron. 1994; 13(5): 1083-1097.

9. Newman MP, Feron F, Mackay-Sim A. Growth factor regulation of neurogenesis in adult olfactory epithelium. Neuroscience. 2000; 99(2): 343-350.

10. Isoyama K, Nagata H, Shino $Y$, et al. Effects of adenoviral vector-mediated BDNF expression on the bulbectomy-induced apoptosis of olfactory receptor neurons. Brain Res Mol Brain Res. 2004; 129(1-2): 88-95.

11. Blomqvist EH, Lundblad L, Bergstedt $H$, Stjarne P. Placebo-controlled, randomized, double-blind study evaluating the efficacy of fluticasone propionate nasal spray for the treatment of patients with hyposmia/anosmia. Acta oto-laryngologica. 2003; 123(7): 862-868.

12. Mott $A E$, Cain WS, Lafreniere D, Leonard G, Gent JF, Frank ME. Topical corticosteroid treatment of anosmia associated with nasal and sinus disease. Arch Otolaryngol Head Neck Surg. 1997; 123(4): 367-372.

13. Seiden AM, Duncan HJ. The diagno- sis of a conductive olfactory loss. The Laryngoscope. 2001; 111(1): 9-14.

14. Heilmann S, Huettenbrink KB, Hummel T. Local and systemic administration of corticosteroids in the treatment of olfactory loss. Am J Rhinol. 2004; 18(1): 29-33.

15. Goldstein BJ, Fang H, Youngentob SL, Schwob JE. Transplantation of multipotent progenitors from the adult olfactory epithelium. Neuroreport. 1998; 9(7): 1611-1617.

16. Jang W, Lambropoulos J, Woo JK, Peluso CE, Schwob JE. Maintaining epitheliopoietic potency when culturing olfactory progenitors. Exp Neurol. 2008; 214(1): 25-36.

17. Kim IY, Seo SJ, Moon HS, et al. Chitosan and its derivatives for tissue engineering applications.Biotechnol Adv. 2008; 26(1): 1-21.

18. Hsueh YY, Chang YJ, Huang TC, et al. Functional recoveries of sciatic nerve regeneration by combining chitosan-coated conduit and neurosphere cells induced from adipose-derived stem cells. Biomaterials. 2014; 35(7): 2234-2244.

19. Hung $\mathrm{CH}$, Lin YL, Young $\mathrm{TH}$. The effect of chitosan and PVDF substrates on the behavior of embryonic rat cerebral cortical stem cells. Biomaterials. 2006; 27(25): 4461-4469.

20. Lin SJ, Jee SH, Hsaio WC, Lee SJ, Young TH. Formation of melanocyte spheroids on the chitosan-coated surface. Biomaterials. 2005; 26(12): 1413-1422.

21. Krolewski RC, Jang W, Schwob JE. The generation of olfactory epithelial neurospheres in vitro predicts engraftment capacity following transplantation in vivo. Exp Neurol. 2011; 229(2): 308-323.

22. Li ST, Young TH, Lin CF, Huang TW. Promotion of olfactory receptor neuron differentiation of olfactory neuroepithelial cells using chitosan solution. Am J Rhinol Allergy. 2017 Sep 1;31(5):289-292.

23. Micholt E, Jans D, Callewaert G, Bartic C, Lammertyn J, Nicolai B. Primary culture of embryonic rat olfactory receptor neurons. In Vitro Cell Dev Biol Anim. 2012 Dec;48(10):650-9.

24. Sakano H. Neural map formation in the mouse olfactory system. Neuron. 2010; 67(4): 530-542.

25. Carter LA, MacDonald JL, Roskams AJ. Olfactory horizontal basal cells demonstrate a conserved multipotent progenitor phenotype. J Neurosci. 2004; 24(25): 5670-5683.

26. Calof AL, Chikaraishi DM. Analysis of neurogenesis in a mammalian neuroepithelium: proliferation and differentiation of an olfactory neuron precursor in vitro. Neuron. 1989; 3(1): 115-127.

27. Wei $H$, Lang MF, Jiang $X$. Calretinin is expressed in the intermediate cells during olfactory receptor neuron development. Neurosci Lett. 2013; 542: 42-46.

28. Kafitz KW, Greer CA. Role of laminin in axonal extension from olfactory receptor cells. J Neurobiol. 1997; 32(3): 298-310.

29. Luppi B, Bigucci F, Cerchiara T, Zecchi V. Chitosan-based hydrogels for nasal drug delivery: from inserts to nanoparticles. Expert Opin Drug Deliv. 2010; 7(7): 811-828.

30. Huang TW, Young YH, Cheng PW, Chan $\mathrm{YH}$, Young $\mathrm{TH}$. Culture of nasal epithelial cells using chitosan-based membranes. Laryngoscope. 2009; 119(10): 2066-2070.

31. Holbrook EH, Szumowski KE, Schwob JE. An immunochemical, ultrastructural, and developmental characterization of the horizontal basal cells of rat olfactory epithelium. J Comp Neurol. 1995; 363(1): 129-146.

Tsung-Wei Huang

Department of Electrical Engineering

College of Electrical and Communica-

tion Engineering

Yuan Ze University

Department of Otolaryngology

Far Eastern Memorial Hospital

21, Section 2, Nan-Ya South Road,

Pan Chiao 220

Taipei

Taiwan

Tel: +886289667000 ext 1513

Fax: + 886229579505

E-mail: huangtw28@gmail.com 